\section{ISOLATION AND STRUCTURES OF MONO- AND DI-DEACETYL CHROMOMYCIN ANTIBIOTICS 02-3D AND 02-3G FROM STREPTOMYCES AVELLANEUS}

\author{
Tsuyoshi Kawano, Tomomi Hidaka, \\ KazUo Furihata, Junichiro MochizUKI ${ }^{\dagger}$, \\ HrRoshi Nakayama, YoIChI Hayakawa \\ and Haruo Seto
}

Institute of Applied Microbiology, University of Tokyo, Bunkyo-ku, Tokyo 113, Japan

†Pharmaceutical Laboratory, Kirin Brewery Co., Ltd., Miyahara, Takasaki, Gunma 370-12, Japan

(Received for publication July 26, 1989)

During the course of a screening program for new antitumor antibiotics from microorganisms using a unique assay system, we have found that Streptomyces avellaneus $02-3$ produced new chromomycin antibiotics, $02-3 \mathrm{D}$ and $02-3 \mathrm{G}$ in addition to several known related antibiotics. In this paper, we wish to report the isolation and structural studies of $02-3 \mathrm{D}$ and $02-3 \mathrm{G}$.

We utilized the phenomenon of DNA intercalation as an assay system. A sample was incubated with $100 \mathrm{ng}$ of pBR322 $\mathrm{ccc}$ form DNA for 30 minutes at $37^{\circ} \mathrm{C}$ and then subjected to $0.7 \%$ agarose gel electrophoresis. Concentrations of actinomycins as low as $0.001 \mu \mathrm{g} / \mathrm{ml}$ were used as a positive control. Decrease in motility of the plasmid DNA in the presence of test samples was judged as a positive result.

The stock culture of $S$. avellaneus $02-3$ was inoculated into a seed medium (starch $1.0 \%$, Polypepton $1.0 \%$, molasses $1.0 \%$ and meat extract $1.0 \%$ ) in a $500-\mathrm{ml}$ Erlenmeyer flask and incubated at $27^{\circ} \mathrm{C}$ for 4 days. The seed culture $(100 \mathrm{ml})$ was transferred to 10 liters of a production medium (glycerol $0.8 \%$, starch $0.8 \%$, soybean meal $0.3 \%$, fish meal $0.8 \%$ and $\mathrm{CaCO}_{3} 0.2 \%$ ) in $500-\mathrm{ml}$ Erlenmeyer flasks and incubated at $27^{\circ} \mathrm{C}$ for 3 days.

A culture filtrate ( 9.5 liters) was adjusted to $\mathrm{pH} 2.0$ with $6 \mathrm{~N} \mathrm{HCl}$, passed through a column of Diaion HP-20, washed with $50 \%$ aq $\mathrm{MeOH}$ and eluted with $\mathrm{MeOH}$. The active fraction was concentrated under reduced pressure to 1 liter and the aqueous residue was extracted with EtOAc. The separated organic layer was evaporated, chromatographed on silica gel and developed with $\mathrm{CHCl}_{3}-\mathrm{MeOH}$ systems (50:1 and $25: 1$ ) containing $1 \%$ volume of $\mathrm{AcOH}$ to give two active fractions. The former fraction was concentrated and applied to preparative silica gel TLC using $\mathrm{CHCl}_{3}-\mathrm{MeOH}(10: 1)$ added with $1 \%$ $\mathrm{AcOH}$. This procedure enabled us to separate a new active compound (02-3D) from chromomycins $\mathrm{A}_{2}$ and $\mathbf{A}_{3}$, deacetylchromomycin $\mathbf{A}_{3}$, aburamycin $C$ and olivomycin $C$. The band of $R f$ value 0.45 corresponding to $02-3 \mathrm{D}$ was extracted with $\mathrm{MeOH}$, concentrated and chromatographed on a Sephadex LH-20 column eluted with $\mathrm{MeOH}$. The active fraction was concentrated and finally applied to preparative HPLC. The conditions were as follows: Senshu Pak ODS-5251-N $(2 \times 25 \mathrm{~cm})$ column; solvent $\mathrm{CH}_{3} \mathrm{CN}-\mathrm{H}_{2} \mathrm{O}(2: 3)$ containing $1 \% \mathrm{AcOH}$; flow rate $9 \mathrm{ml} /$ minute; detection by $\mathrm{UV}$ absorption at $280 \mathrm{~nm} .02-3 \mathrm{D}$ was eluted at 28 minutes, concentrated to $1 / 3$ volume, extracted with EtOAc, concentrated and subjected to Sephadex LH-20 column chromatography developed with $\mathrm{MeOH}$. After concentration, $02-3 \mathrm{D}$ was obtained as a yellow powder (10 mg).

The latter fraction was concentrated and applied to preparative silica gel TLC using $\mathrm{CHCl}_{3}-\mathrm{MeOH}$ (6:1) added with $1 \% \mathrm{AcOH}$. The band of $\mathrm{Rf}$ value 0.40 was extracted with $\mathrm{MeOH}$, concentrated and chromatographed on a Sephadex LH-20 column eluted with $\mathrm{MeOH}$. The active fraction was concentrated and finally applied to preparative HPLC. The condition were almost the same as described above except for the composition of the solvent being changed to $\mathrm{CH}_{3} \mathrm{CN}-\mathrm{H}_{2} \mathrm{O}(35: 65)$ containing $1 \% \mathrm{AcOH}$. 02-3G was eluted at 35 minutes. After the same procedure as just described, $15 \mathrm{mg}$ of $02-3 \mathrm{G}$ was obtained as a yellow powder.

The physico-chemical properties of $02-3 \mathrm{D}$ and 02-3G were as follows. 02-3D: MP $180 \sim 184^{\circ} \mathrm{C}$; $[\alpha]_{\mathrm{D}}^{20}$ $+55.6^{\circ}$ (c $\left.0.1, \mathrm{MeOH}\right) ; \mathrm{UV} \lambda_{\max }^{\mathrm{MeOH}} \mathrm{nm}(\varepsilon) 230$ $(26,900), 280(50,800), 318(9,100), 331(7,100)$, $414(10,600)$; IR $v_{\max }(\mathrm{KBr}) \mathrm{cm}^{-1} 3420,1722,1629$; FAB-MS $m / z \quad 1,139(\mathrm{M}-\mathrm{H}$, corresponding to the molecular formula $\mathrm{C}_{55} \mathrm{H}_{80} \mathrm{O}_{25}$ ).

02-3G: MP $181 \sim 186^{\circ} \mathrm{C}(\mathrm{dec}) ;[\alpha]_{\mathrm{D}}^{20}+21.1^{\circ}(\mathrm{c} 0.1$, $\mathrm{MeOH}) ; \mathrm{UV} \lambda_{\max }^{\mathrm{MeOH}} \mathrm{nm}(\varepsilon) 230(26,600), 280(46,800)$, $318(9,000), 331(7,600), 416(9,800)$; IR $v_{\max }(\mathrm{KBr})$ $\mathrm{cm}^{-1} 3410,1718,1625$; FAB-MS m/z 1,097 (M-H, corresponding to the molecular formula $\mathrm{C}_{53} \mathrm{H}_{78} \mathrm{O}_{24}$ ), analysis; Calcd: C 57.91, $\mathrm{H} 7.15, \mathrm{O}$ 34.93, Found: C 57.99, H 7.23, O 34.98 .

The structures of $02-3 \mathrm{D}$ and $02-3 \mathrm{G}$ were finally determined by ${ }^{1} \mathrm{H}$ and ${ }^{13} \mathrm{C}$ NMR spectral analyses and by comparison with related compounds, chromomycin $\mathrm{A}_{3}{ }^{1 \sim 3)}$ and $\mathrm{K}-2^{4,5)}$ as shown in Fig. 1.

The procedure for the structural determination of 02-3D was as follows: Upon comparison of ${ }^{13} \mathrm{C}$ 
Table $1 .{ }^{13} \mathrm{C}$ NMR data of $02-3 \mathrm{D}, 02-3 \mathrm{G}$ and related compounds.

\begin{tabular}{|c|c|c|c|c|}
\hline & $\begin{array}{c}02-3 \mathrm{D} \\
\left(\mathrm{CDCl}_{3}\right)\end{array}$ & $\begin{array}{c}02-3 \mathrm{G} \\
\left(\mathrm{CD}_{3} \mathrm{COOD}\right)\end{array}$ & $\begin{array}{c}\mathrm{K}-2 \\
\left(\mathrm{CDCl}_{3}-\mathrm{CD}_{3} \mathrm{OD}\right)\end{array}$ & $\begin{array}{l}\text { Chromomycin } \mathrm{A}_{3} \\
\left(\mathrm{CDCl}_{3}-\mathrm{CD}_{3} \mathrm{OD}\right)\end{array}$ \\
\hline \multicolumn{5}{|l|}{ Aglycone } \\
\hline C-1 & 202.5 & 203.7 & 203.8 & 202.7 \\
\hline C-2 & 77.2 & 77.8 & 77.8 & 77.0 \\
\hline $\mathrm{C}-3$ & 43.1 & 43.7 & 42.4 & 43.4 \\
\hline C-4 & 27.2 & 27.9 & 27.7 & 27.3 \\
\hline C-5 & 101.3 & 102.3 & 101.7 & 101.6 \\
\hline C-6 & 159.9 & 160.3 & 160.0 & 160.0 \\
\hline C-7 & 111.7 & 112.2 & 111.6 & 111.9 \\
\hline C-8 & 164.8 & 165.0 & 165.5 & 165.8 \\
\hline C-9 & 156.2 & 156.6 & 156.5 & 156.6 \\
\hline C-10 & 117.3 & 118.1 & 117.2 & 117.4 \\
\hline$C-4 a$ & 135.4 & 136.3 & 136.7 & 135.4 \\
\hline$C-8 a$ & 108.5 & $108.7^{\mathrm{a}}$ & $108.5^{\mathrm{a}}$ & 108.6 \\
\hline $\mathrm{C}-9 \mathrm{a}$ & 108.5 & $109.0^{\mathrm{a}}$ & $108.8^{\mathrm{a}}$ & 108.6 \\
\hline $\mathrm{C}-10 \mathrm{a}$ & 138.8 & 139.4 & 139.0 & 138.9 \\
\hline 7- $\mathrm{CH}_{3}$ & 8.2 & 8.5 & 8.2 & 8.3 \\
\hline$C-1^{\prime}$ & 81.8 & 83.1 & $78.6^{\mathrm{b}}$ & 82.4 \\
\hline $\mathrm{C}-2^{\prime}$ & 211.8 & 212.3 & $72.2^{\mathrm{b}}$ & 211.9 \\
\hline$C-3^{\prime}$ & 78.8 & 79.3 & $74.0^{\mathbf{b}}$ & 79.0 \\
\hline C- $4^{\prime}$ & 68.3 & 69.1 & $70.2^{\mathrm{b}}$ & 68.4 \\
\hline C- $5^{\prime}$ & 19.7 & 20.0 & 19.7 & 19.9 \\
\hline $1^{\prime}-\mathrm{OCH}_{3}$ & 59.3 & 59.9 & 60.6 & 59.5 \\
\hline \multicolumn{5}{|l|}{ A-sugar } \\
\hline C-1 & 97.7 & 98.4 & 98.1 & 97.9 \\
\hline C-2 & 32.5 & 33.1 & 32.7 & 33.3 \\
\hline $\mathrm{C}-3$ & 73.3 & 73.8 & 73.4 & 70.6 \\
\hline $\mathrm{C}-4$ & 66.8 & 68.2 & 67.3 & 68.3 \\
\hline C-5 & 71.5 & 72.0 & 71.6 & 70.2 \\
\hline C-6 & 16.9 & 17.1 & 16.9 & 16.9 \\
\hline $\mathrm{CH}_{3} \mathrm{CO}$ & - & - & - & 20.7 \\
\hline $\mathrm{CH}_{3} \mathrm{CO}$ & - & - & - & 171.7 \\
\hline \multicolumn{5}{|l|}{ B-sugar } \\
\hline C-1 & 96.0 & 96.6 & 96.1 & 95.8 \\
\hline $\mathrm{C}-2$ & 33.2 & 33.5 & 33.5 & 33.3 \\
\hline $\mathrm{C}-3$ & 66.4 & 67.3 & 66.7 & 66.6 \\
\hline $\mathrm{C}-4$ & 81.8 & 82.5 & 82.1 & 82.1 \\
\hline C-5 & 67.3 & 68.2 & 67.5 & 67.6 \\
\hline C-6 & 17.1 & 17.2 & 17.1 & 17.2 \\
\hline $\mathrm{OCH}_{3}$ & 62.1 & 62.3 & 62.1 & 62.1 \\
\hline \multicolumn{5}{|l|}{ C-sugar } \\
\hline C-1 & 100.8 & 101.7 & 100.8 & 101.0 \\
\hline $\mathrm{C}-2$ & 37.6 & 38.2 & 37.7 & 37.8 \\
\hline C-3 & 81.8 & 82.3 & 81.9 & 81.9 \\
\hline C-4 & $75.5^{\mathrm{a}}$ & 76.5 & 75.8 & 75.8 \\
\hline $\mathrm{C}-5$ & $72.4^{b}$ & 72.7 & 72.6 & 72.7 \\
\hline C-6 & $17.9^{\mathrm{c}}$ & $18.5^{\mathrm{c}}$ & 18.2 & 18.1 \\
\hline \multicolumn{5}{|l|}{ D-sugar } \\
\hline C-1 & 99.7 & 100.5 & 99.8 & 99.8 \\
\hline $\mathrm{C}-2$ & 36.9 & 37.3 & 37.2 & 37.2 \\
\hline $\mathrm{C}-3$ & 77.7 & 77.8 & 78.6 & 78.4 \\
\hline C-4 & $75.1^{\mathbf{a}}$ & 76.2 & 75.6 & 75.5 \\
\hline C-5 & $72.7^{\mathrm{b}}$ & 73.1 & 72.8 & 72.9 \\
\hline C-6 & $17.9^{c}$ & $18.2^{\mathrm{c}}$ & 18.0 & 18.0 \\
\hline
\end{tabular}


Table 1. (Continued)

\begin{tabular}{ccccc}
\hline & $\begin{array}{c}02-3 \mathrm{D} \\
\left(\mathrm{CDCl}_{3}\right)\end{array}$ & $\begin{array}{c}02-3 \mathrm{G} \\
\left(\mathrm{CD}_{3} \mathrm{COOD}\right)\end{array}$ & $\begin{array}{c}\mathrm{K}-2 \\
\left(\mathrm{CDCl}_{3}-\mathrm{CD}_{3} \mathrm{OD}\right)\end{array}$ & $\begin{array}{c}\mathrm{Chromomycin}_{3} \\
\left(\mathrm{CDCl}_{3}-\mathrm{CD}_{3} \mathrm{OD}\right)\end{array}$ \\
\hline E-sugar & & & & \\
$\mathrm{C}-1$ & 95.7 & 96.2 & 96.5 & 96.1 \\
$\mathrm{C}-2$ & 43.7 & 43.9 & 43.6 & 44.0 \\
$\mathrm{C}-3$ & 70.4 & 72.0 & 71.5 & 70.6 \\
$\mathrm{C}-4$ & 79.5 & 80.1 & 79.6 & 79.8 \\
$\mathrm{C}-5$ & 66.8 & 68.6 & 68.4 & 67.1 \\
$\mathrm{C}-6$ & $18.1^{\mathrm{c}}$ & 18.1 & 18.0 & 17.9 \\
$3-\mathrm{CH}_{3}$ & 22.7 & 22.2 & 22.1 & 23.0 \\
$\mathrm{CH}_{3} \mathrm{CO}$ & 21.0 & - & - & 20.9 \\
$\mathrm{CH}_{3} \mathrm{CO}$ & 171.6 & - & - & 171.6 \\
\hline
\end{tabular}

$a, b, c$ Assignments in any column may be exchanged.

Fig. 1. Structures of $02-3 D, 02-3 G$ and their related compounds.

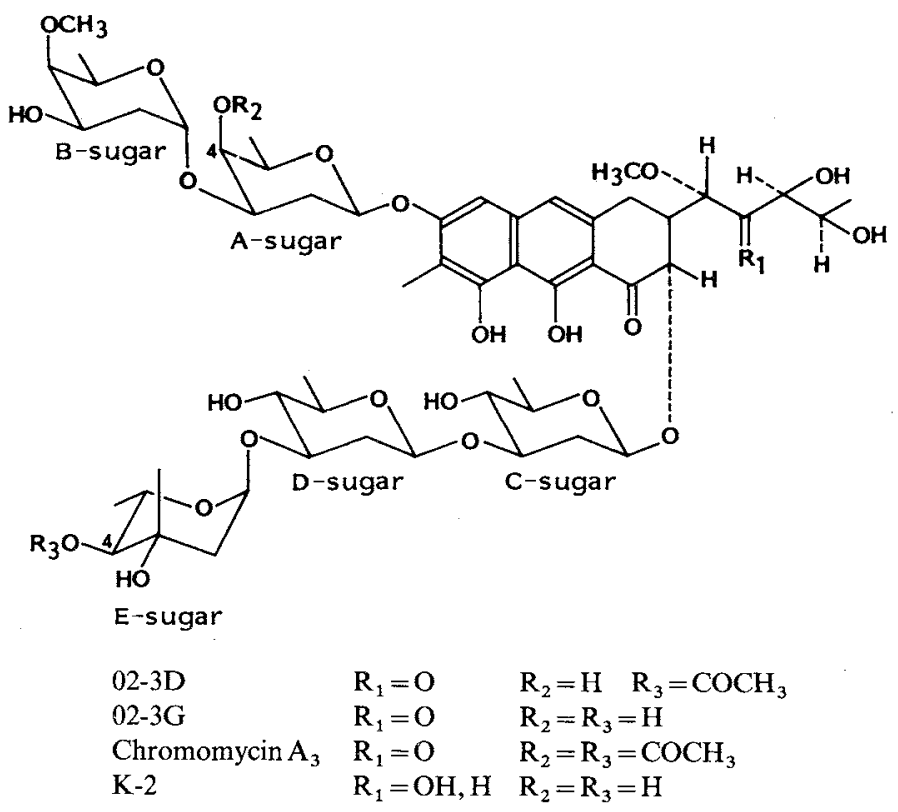

NMR spectral data (Table 1), 55 out of the 57 signals of chromomycin $A_{3}$ were identified in the spectrum of 02-3D. Two signals absent in $02-3 \mathrm{D}$ were ascribed to one acetyl group. This result was supported by the decrease in molecular weight of $02-3 \mathrm{D}$ by 42 units compared to chromomycin $\mathrm{A}_{3}$. Furthermore, the signal derived from $4 \mathrm{~A}-\mathrm{H}$, which was found at $5.15 \mathrm{ppm}$ in the ${ }^{1} \mathrm{H}$ NMR spectrum of chromomycin $A_{3}$, was found at $3.73 \mathrm{ppm}$ with $02-3 \mathrm{D}$. The assignments of the key signals were made by ${ }^{13} \mathrm{C}$ ${ }^{1} \mathrm{H} \mathrm{COSY}^{6)},{ }^{1} \mathrm{H}-{ }^{1} \mathrm{H} \operatorname{COSY}^{7,8)}$ and homonuclear Hartman-Hahn (HOHAHA) ${ }^{9)}$ (data not shown). These results clearly showed that $02-3 \mathrm{D}$ is the Asugar deacetyl derivative of chromomycin $\mathrm{A}_{3}$.

The structure of $02-3 \mathrm{G}$ was determined as follows:
Comparison of the ${ }^{13} \mathrm{C}$ NMR spectral data (Table 1) showed that 53 signals of $02-3 \mathrm{G}$ were identified with those of chromomycin $\mathrm{A}_{3}$ and that four absent signals were assignable to two acetyl groups. In. addition, signals derived from the sugars in $02-3 \mathrm{G}$ coincided with those of $\mathrm{K}-2$ which is a dideacetyldihydro derivative of chromomycin $\mathrm{A}_{3}$. These results showed that $02-3 \mathrm{G}$ is dideacetyl chromomycin $\mathrm{A}_{3}$.

02-3D and $02-3 \mathrm{G}$ inhibited the growth of P388 murine leukemia cells at the $\mathrm{IC}_{50}$ value of 12 and $0.60 \mu \mathrm{g} / \mathrm{ml}$, respectively. On the other hand, they could be detected at concentrations of 0.1 and $0.01 \mu \mathrm{g} / \mathrm{ml}$, respectively, by our assay system using intercalation with DNA. Consequently, this assay system is a sensitive one and may enable the 
detection of compounds which have not been discovered by other assay systems.

\section{Acknowledgments}

This work was supported in part by a Grant-in-Aid for Cancer Research, The Ministry of Education, Science and Culture, Japan.

\section{References}

1) Mizuno, K.: Studies on chromomycin. IV. Chromomycin $\mathrm{A}_{3}$ and its derivatives. J. Antibiotics, Ser. A 16: $22 \sim 39,1963$

2) THIEM, J. \& B. MEYER: Studies on the structure of chromomycin $\mathrm{A}_{3}$ by ${ }^{1} \mathrm{H}$ and ${ }^{13} \mathrm{C}$ nuclear magnetic resonance spectroscopy. J. Chem. Soc. Perkin Trans. II 1979: $1331 \sim 1338,1979$

3) Riccio, R. \& K. NAKANISHI: Circular dichroic method for determining the position of glycosidic linkage of deoxysugar moieties of antitumor antibiotic chromomycin $A_{3}$. J. Org. Chem. 47: 4589 4952, 1982

4) Koenuma, M.; N. Uchida, K. Yamaguchi, Y.
Kawamura \& K. Matsumoto: New aureolic acid antibiotics. I. Screening, isolation, characterization and biological properties. J. Antibiotics 41: 45 52, 1988

5) Yoshimura, Y.; M. Koenuma, K. Matsumoto, K. TORI \& Y. TeruI: NMR studies of chromomycins, olivomycins, and their derivatives. J. Antibiotics 41 : $53 \sim 67,1988$

6) BAX, A. \& G. Morris: An improved method for heteronuclear chemical shift correlation by twodimensional NMR. J. Magn. Reson. 42: $501 \sim 505$, 1981

7) Aue, W. P.; E. Bartholde \& R. R. ERnst: Two-dimensional spectroscopy. Application to nuclear magnetic resonance. J. Chem. Phys. 64: 2229 2246, 1976

8) Nagayama, K.; A. Kumar, K. Wuthrich \& R. R. ERNST: Experimental techniques of two-dimensional correlated spectroscopy. J. Magn. Reson. 40: $321 \sim 325,1980$

9) Davis, D. G. \& A. BAx: Assignment of complex ${ }^{1} \mathrm{H}$ NMR spectra via two-dimensional homonuclear Hartmann-Hahn spectroscopy. J. Am. Chem. Soc. 107: $2820 \sim 2821,1985$ 